\title{
A Routing Protocol Based on Both of Density Variation and Distance-Aware for WSNs
}

\author{
Dongmei XING ${ }^{1}$ \\ Department of Mathematics, Nanchang University, Nanchang, China
}

\begin{abstract}
A hierarchical routing algorithm for wireless sensor networks (WSNs) is discussed. We select cluster heads according to related distances and residual energy. Both effects of the number of nodes dissipated and the energy consumption act on propagation distances. In addition, the related density effects on the propagation distance. We Define comprehensive influence factor and propagation influence factor, adjust the initial probability of nodes participating in cluster heads' election, make propagation distances of nodes gradually increase within a certain range. Simulation results show that both cluster heads and failure nodes are evenly distributed in the whole sensor network. The residual energy of nodes are balanced inter the living nodes, which extends the survival time of the network. The routing algorithm we have designed has the characteristics of better balanced energy consumption.
\end{abstract}

Keywords sensor, routing algorithms, density, energy reduction, failure factors.

\section{Introduction}

WSNs are made up of a lot of sensors with low energy. Sensors whose bodies are small can live in all sorts of environment and have several functions such as calculation, store and forward. The convenience of being carried and used let WSNs have extensive application prospect. Sensors have their own disadvantages, e.g. their limited energies, it is necessary to avoid their disadvantage. It is not convenient to add energy if any sensor has or is about to be dissipated in some application environments. When the data are forwarded in WSNs, the appropriate routing algorithm has to save energy and let the energy be consumed uniformly as much as possible. In order to extend the lifetime of WSNs, we should ensure living sensors with higher residual energy or being distributed uniformly in the network.

For the sake of convenience, sensors are named as nodes in the following, the node that finally receives data is the base station (or the sink node), and the node that sends data is called as source node. In a larger layout range, the geometric distances between the source nodes and the sink node are longer, it is not in one-hop to transport data, instead of multi-hop forwarding data. The nodes with same initial energy are uniformly distributed, but along data transmission, different nodes consume different energies and some nodes may fail in advance. The living nodes may be no longer uniformly distributed, and the residual energy of living nodes may be different. Suppose the data

\footnotetext{
${ }^{1}$ Corresponding Author, email: dongdongsh2000@sina.com.
} 
is transported in distributed way in the network. With some admissible delay, the same received-data needs to be aggregated before being forwarded to the sink node. Data may be aggregated at certain relay nodes, and then be forwarded to the sink node. The hierarchical routing protocols mainly involve two phases, namely the cluster construction phase and the data transmission in the stable phase. A clustering can be divided into five steps, namely, computing the optimal number of cluster heads, selecting the optimal cluster heads, calculation of clusters' radius, constructing clusters, processing some special nodes (such as isolated nodes).

We designed a hierarchical route algorithm, the basic steps and requirements are described as follows.

1. Initial step. Set two influence factors on cluster heads' election and propagation radius. Initialize parameters.

2. Using residual energy, density change rate and related radio radius to adjust the probability of participating in the competition of cluster heads and cluster radius.

3. Selecting cluster heads in distributed way.

4. After all nodes have been joined in corresponding clusters, first special nodes (such as isolated nodes) would be processed, second the data is transported and forwarded. It is supposed that the received data could be aggregated before the data forwarded to another cluster head or to the sink node.

5. Supposed that conflicts are avoided during data transmission and information will not be lost.

\section{Related Literatures}

If sensors with homogeneity are arranged into a flat topology uniformly, the transmission range of WSNs is consistent with the coverage range if the propagation radius of the sensor meets certain conditions [1][2].

LEACH [3] is the first hierarchical clustering routing protocol. Many improved algorithms on WSNs have appeared [4-7] based on LEACH. LEACH protocol specifies the expected percent of the cluster heads and gives a threshold for each node to be selected as a cluster head. If the random number $p(n)$ assigned to node $n$ is less than threshold $T(n)$, node $n$ is selected as the cluster head, otherwise it is a noncluster head node. Non-cluster-head nodes join in someone cluster head according to sensing strength. In each cluster, non-cluster-head nodes transmit data to their cluster heads in a single hop. Cluster heads send received data (after received data being aggregated) to the sink node in one-hop. Data forwarded between cluster heads and BS is completed in a one-hop mode, and the cluster head election is randomness and equilibrium.

Hierarchical cluster protocol HEED [8] takes into both the communication overhead and residual energy of each node. Data from source nodes to the sink node can be transported by multiple hops, and the relay nodes passing through are all clusterhead nodes. When a cluster head is selected by HEED, both primary and secondary factors are involved. The main factor is the percentage of the current residual energy $E_{r}$ to the initial energy $E_{m}$ of each node; the secondary factor is the communication energy consumption between the nodes in the cluster. Any cluster is constructed in HEED by three steps: initialization, loop step and final step. 
Initialization. Calculate the neighboring sets, compute the minimum average communication energy consumption of each cluster (the neighboring set of a certain node). Set the initial probability $\mathrm{CH}_{\text {prob }}$ that any node is selected as a cluster head.

$$
\mathrm{CH}_{\text {prob }}=\max \left(C_{p} \times E_{r} / E_{m}, p_{\min }\right),
$$

where $C_{p}$ is the expected percentage of the cluster heads in the WSNs, $p_{\min }$ is the minimum probability that any node becomes a cluster-head node, which is mainly used to ensure the convergence of HEED. The initial probability $\mathrm{CH}_{\text {prob }}$ changes with the network operation and residual energy of the node, which is the final probability that the node is selected as the cluster head.

Loop step. The main task is to select cluster heads from cluster heads' set $S_{C H}$, where cluster heads are divided into two sorts that are temporary cluster heads and final cluster heads.

In the final stage, each node determines its final state (either a member node of a cluster or a final cluster head). If the node is an isolated node, it is declared to be a cluster-head node.

Kuang zhejun [9] proposed a clustering algorithm REEM (A role, energy efficient, membership clustering) based on role membership in static network in order to eliminate "energy hole". REEM considers roles and relationships. Relationships among nodes are divided into member relationship and non-member relationship. Nodes' roles are divided into three sorts that are dominant node ${ }^{2}$, relay node and source node according to their residual energy. Different roles transform identity by their own energy (residual energy of dominant node $>$ residual energy of relay node $>$ residual energy of source node). Unselected relay nodes are in dormant state. In REEM algorithm, dominant nodes send data to BS in one-hop, but the data is sent and forwarded from the source to a dominant node through an intermediate path composed of relay nodes. Prasenjit Chanak et al. [10] studied WSNs with uniform distribution, homogeneity and static layout. By the characteristics of the sensor network based on tasks, they proposed the problem of reducing the energy hole in the network in according to load management. The network is divided into several clusters whose scale can be determined [4].The algorithm of load management mode [4] is completed in two steps. The first step selects the cluster head from the perspective of energy saving and complete the cluster construction. The second step would select the appropriate route path for the purpose of load balancing. Routing strategy considers the energy consumption of receiving and forwarding data. Both of clustering and selecting cluster heads are completed according to the energy consumption of the nodes, which is not completed in a probabilistic way like LEACH, HEED and other similar protocols.

The combination of optimization algorithms and cluster head selection strategies in hierarchical protocols is also a method to study wireless network routing algorithms. Tarunpreet Bhatia et al. [11] proposed routing algorithm GADA-LEACH (Genetic Algorithm based Distance-Aware LEACH) in wireless sensor networks by using genetic algorithm. First, LEACH protocol is used to select the initial cluster head. Then, define the fitness function according to three factors. Genetic algorithm is used to optimize the primary cluster head. An energy efficient clustering and routing method GECR (Genetic algorithm based Energy efficient Clustering and Routing approach) is

${ }^{2}$ For the sake of distinction, sink nodes in [9] is called dominant nodes in this paper. 
designed by using genetic algorithm [12]. K. Thangaramya et al.[13] used fuzzy neural network to design an energy-sensitive clustering protocol.

In addition, some routing protocols consider other performance indexes of sensor networks and design corresponding hierarchical routing protocols. Ngoctu Nguyen et al. [14] designed the routing algorithm to maximize the utility of data aggregation and constructed a virtual aggregation tree by equilibrium adjustment in order to minimize the load consumption. By the tree, the data is received and forwarded to balance the energy consumption, so as to reduce the energy hole. Anuradha Pughat et al. [15] used the dynamic energy management model to analyze the performances. With the goal of minimizing energy consumption, Mohit Sajwan et al. [16] designed optimization algorithms of selecting multi-path routing or single-hop routing. Ansam Ennaciri et al. [17] designed the routing strategy from the perspective of load balancing with the goal of improving service quality. Surjit Singh etc. [18,19] discussed performance evaluation by optimization techniques or DMS (Dynamic Modulation Scaling).

\section{Relevant Concepts}

\subsection{Radio Model}

We use the First Order Radio Model (figure 1). The Settings of various parameters are shown in table 1 2. The energy consumption of transmitting 1 bit and the energy consumption of receiving 1 bit are respectively denoted as $E_{T x}$ (Transmitter Electronics ), $E_{R x}$ (Receiver Electronics). The energy consumption $E_{T x}(k, d)$, which is generated by a packet with $k$ bits ( $\mathrm{k}$ bits in a packet) transmitted to the target node at distance $d$, is consists of the energy consumption both of data transmission and data amplification (respectively denoted as $E_{T x}(k), E_{\text {amp }}(k, d)$ ). The energy consumption of the packet received is denoted as $E_{R x}(k)$.

TABLE 1 Radio model Parameters

\begin{tabular}{c|l|l|l|c}
\hline Type & Parameter & Value & Parameter & Value \\
\hline Radio model & $E_{\text {elec }}, E_{T x}, E_{R x}$ & $50 \mathrm{~nJ} / \mathrm{bit}$ & $E_{\text {fusion }}$ & $E_{D A}=5 n J /$ bit $/$ signal \\
& $e_{f s}$ & $10 \mathrm{pJ} / \mathrm{bit} / \mathrm{m}^{2}$ & Threshold distance $\left(d_{0}\right)$ & $d_{0}=75 m<\sqrt{e_{f s} / e_{\text {amp }}}$ \\
& $e_{a m p}$ & $0.0013 \mathrm{pJ} / \mathrm{bit} / \mathrm{m}^{4}$ & & \\
\hline
\end{tabular}

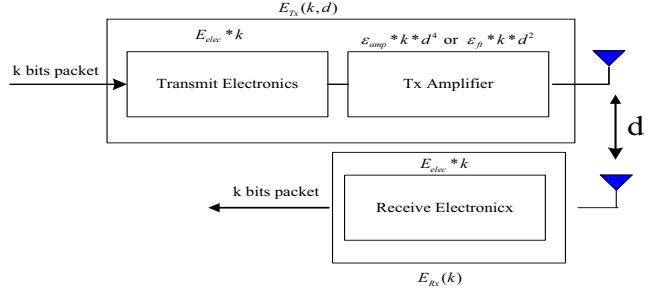

Fig 1 The First Order Radio Model

Computing $E_{T x}(k, d)[3,4]$ as follows.

$$
E_{R x}(k)=E_{T x}(k, d)=E_{\text {elec }} * k,
$$




$$
\begin{aligned}
& E_{T x}(k, d)=E_{T x}(k)+E_{\text {amp }}(k, d) \\
& =\left\{\begin{array}{l}
E_{\text {elec }} * k+\varepsilon_{f s} * k * d^{2}, \quad d<d_{0} \\
E_{\text {elec }} * k+\varepsilon_{\text {amp }} * k * d^{4}, d \geq d_{0}
\end{array}\right.
\end{aligned}
$$

\subsection{Influence Factors Design and Parameter Description}

Suppose that $n_{m}$ sensors with initial energy $E_{m}$ are uniformly placed in a rectangular area .The remaining energy of a node at a certain time and the number of nodes alive in the network are denoted as $E_{r}, n_{\text {alive }}$, respectively. The initial probability of a node becoming a cluster head is defined as follows.

$$
\mathrm{CH}_{\text {prob }}=\max \left(C_{p} \times E_{r} / E_{m} \times f_{p}, p_{\text {min }}\right)
$$

where $C_{p}$ is the expected percentage of the cluster heads in the network, and $p_{\min }$ is the minimum probability that a node becomes a cluster header. $f_{p}$ is the propagation influence factor with initial value 1 , and its meaning is shown in the following Definition 3. $\mathrm{CH}_{\text {prob }}$ would change with the network operation and the residual energy of the node, which is also the final probability that the node is selected as the cluster head.

Cluster head election and radio radius usually change with the change of network energy. In the process of data transmission and forwarding, energy consumption is generally uneven. Considering the influence factors from both the whole and the part, we design the energy consumption reduction factor $a_{0}$ and the failure influence factor 1 (denoted as $a_{1}$ ). We also design density impact factor (denoted as $b_{0}$ ) and propagation impact factor $f_{P}$, failure impact factor 2 (denoted as $b_{1}$ ) and synthesis factor $f_{R}$.

Definition 1 Cost factor of energy reduction $a_{0}$ and failure impact factor $1 a_{1}$ are defined as follows.

$$
a_{0}=1+\sum \frac{E_{m}-E_{r}}{n_{\text {alive }} * E_{m}}, a_{1}=1+\frac{n_{m}-n_{\text {alive }}}{n_{m}} .
$$

Definition 2 Density impact factor $b_{0}$ and failure impact factor $2 b_{1}$ are defined as follows.

$$
b_{0}=1-\frac{n_{\text {TnumNbr }}-n_{\text {numNbr }}}{n_{m}}, \quad b_{1}=1+\frac{n_{\text {Talive }_{0}}-n_{\text {alive }_{0}}}{n_{\text {Talive }_{0}}},
$$

where, $n_{\text {TnumNbr }}$ and $n_{\text {numNbr }}$ are expressed respectively the number of neighbor nodes before and after data information transmission in the same round; $n_{\text {Talive }_{0}}$ and $n_{\text {alive }_{0}}$ are respectively the number of living nodes before and after data information transmission in the same round.

Definition 3 (energy consumption and failure) Comprehensive impact factor (denoted as $f_{R}$ ), and propagation impact factor (denoted as $f_{p}$ ) are defined as follows. 


$$
\begin{aligned}
& f_{R}=\left(0.5 * a_{0}+0.5 * a_{1}\right) * b_{1}, \text { noticing } b_{1}=1 \text { (initial), so } f_{R}=1 \text { (initial); } \\
& f_{P}=\left(R_{1} / d\right)^{2}, \text { here } f_{P}=1 \text { (initial), } R_{1}=b_{0} * R,
\end{aligned}
$$

where $R$ is the propagation radius of nodes (each node's $R$ is same in the same round), $d$ denotes the distance from non-cluster-head to cluster-head or cluster-head to BS.

Definition 4 The initial value of the communication transmission radius $R_{0}=\sqrt{X^{2}+Y^{2}} \times \sqrt{\frac{\log n_{m}}{n_{m}}}$, the adjustment formula of the transmission radius is $R=\min \left(f_{R} * R_{0}, d_{0}\right)$, where $d_{0}$ is shown in table 1 .

\subsection{Design Idea}

We believe that if a node consumes more energy in the previous round, it is less competitive in the following cluster-head competition. If the density of nodes has a larger change, the chance of selecting cluster head is smaller. The propagation distance of nodes increases with the increase of the failure node. The initial value of each node's $\mathrm{CH}_{\text {prob }}$ is modified according to both the residual energy and the propagation influence factor before the head election. The comprehensive influence factor is involved in the adjustment of the propagation distance of nodes. If the selected cluster head has no member node or just one member node, it is added to the nearest cluster and become a member of this cluster. Each member node send data to its cluster head by one-hop or two-hop, then cluster heads forward data to the sink node by multi-hop (or one-hop) after data aggregated.

\section{Our routing algorithm is divided into four steps.}

Step 1. Initialization. Set various parameters.

Step 2. Loop stage. Complete the function of cluster heads election and clustering.

Step 3. For nodes not in any cluster, if they are not the final cluster heads, set them as the final cluster head to complete the cluster construction; If they are final cluster heads, complete the cluster construction.

Step 4. Adjustment. A cluster head is isolated or has only one cluster member, then it is added to the nearest cluster and become a cluster member of this cluster. Adjust the member set of this cluster.

\section{Algorithm Strategy and Explanations}

\subsection{Routing Algorithm}

Our routing algorithm is an improvement of HEED routing protocol and named as Alg (in attach). It is briefly described as follows. Firstly, set parameters and define the initial radio radius $\mathrm{R}$. Then select and determine the final cluster head to complete the cluster building operation (steps II IV below). Finally, data transmission is occurred in WSNs. 


\subsection{Theoretical Explanation}

Observation 1: Algorithm Alg is completely distributed and satisfies the conditions listed in the introduction. Any node becomes a cluster head node according to its $\mathrm{CH}_{\text {prob }}$, or become a cluster member node.

The result of Observation can be obtained according to lemma 1 3 below.

Lemma 1 the Alg algorithm will terminate in O(1).

Proof It is proved that Initialize, Finalize and TDMA in Alg can obviously be completed in limited steps, and the corresponding steps in Repeat are similar to those steps in Heed [8], so Alg can be completed in O(1).

Lemma 2 At the end of step III, a node is either a cluster head or a normal node of a cluster (that is a member node and is a non-cluster head). In general, cluster heads are not isolated cluster heads, unless both the number of live nodes and the number of cluster heads are the same one node.

Proof Two cases.

1. If $\mathrm{CH}_{\text {prob }}$ and $\mathrm{CH}_{2}{ }_{\text {prob }}$ of two adjacent nodes is different from each other, $\mathrm{CH} 1_{\text {prob }}$ and $\mathrm{CH} 2_{\text {prob }}$ cannot reach 1 at the same time in step III, then they cannot be selected as cluster-head nodes at the same time.

2. If $\mathrm{CH}_{\text {prob }}$ equals to $\mathrm{CH} 2_{\text {prob }}$, their values reach 1 at the same time, but the probability of this is small. The above result has been shown in [8](reference Lemma2 in [8]).

Lemma 3 Algorithm Alg can be used to select cluster heads in a distributed way. A node is either a cluster head node, or one or two hops can reach a cluster head node, and the cluster heads obtained are evenly distributed in the network range.

Proof Consider and Analysis step IV in Alg. According to using the conclusions of lemma 1, 2 and Lemma5 in [8], the conclusion of Lemma is easy to be drawn.

\subsection{Energy Consumption Calculation}

Energy consumption mainly lies in data transmission, forwarding and data aggregation. In the process of cluster head election, each node is either selected as a cluster head or join to a cluster to become a member node of a non-cluster head. In the process of each node confirming its role, the data sent or received is relatively small. Once the role is determined, the amount of aggregated data, as well as the amount of data sent or received, needs to be measured as the number of packets. There are three cases: energy consumption of one-hop (or two-hop) normal cluster members, energy consumption of cluster heads, and energy consumption of relay nodes (relay nodes refer to cluster head nodes with only one normal cluster member).

Energy consumption can be divided into three cases (in the process of data transmission and forwarding).

Case 1. Compute energy consumption of normal member nodes (non-cluster-head member nodes). Similar to formula equation (1).

Case 2. Isolated cluster heads and clusters with only one cluster member. An isolated cluster head is directly added to another nearby cluster and become its normal cluster member. Processing the clusters with only one normal cluster member, see the following description. In fig 2, Data transmission between isolated sensors and clusters with only one cluster member is expressed. 
1. Isolated cluster head $u$ join to a cluster whose cluster head is $C H . u$ sent $l$ bits to $C H$, the energy consumption is denoted as $E_{u-C H}$ or $\operatorname{Tr}(u)$.

$$
\begin{aligned}
E_{u-C H} & =\operatorname{Tr}(u)=E_{T x}\left(l, d_{u-t o-C H}\right) \\
& =l *\left[E_{\text {elec }}+e_{f s} * d_{u-t o-C H}{ }^{2}\right],
\end{aligned}
$$

2. A cluster $u$ with only one normal cluster member $v$ (here $u$ is the cluster head, re-labeled $u$ as the relay node). $v$ sent $l$ bit to the relay node $u$, then $u$ receives and forwards data to a cluster head $C H$, the energy consumption $E_{v-u-C H}$ is calculated as follows.

$$
\begin{aligned}
E_{v-u-C H} & =\operatorname{Tr}(v)+\operatorname{Re}(u)+2 * l * E_{D A}+\operatorname{Tr}(u) \\
& =l *\left[E_{\text {elec }}+e_{f s} * d_{v-t o-u}{ }^{2}\right]+l * E_{\text {elec }}+2 * l * E_{D A}+\alpha * 2 * l *\left(E_{\text {elec }}+e_{f s} * d_{\text {utoCH }}{ }^{2}\right) \\
& =l * e_{f s} *\left(d_{v t o u}{ }^{2}+2 \alpha *{d_{u t o C H}}^{2}\right)+2 * l * E_{D A}+2 *(1+\alpha) l * E_{\text {elec }},
\end{aligned}
$$

Case 3. Normal cluster-head nodes (whose size is $n_{C H}>2$, where the size of a cluster refers to the number of nodes in the cluster).

Cluster head energy consumption $=$ received data energy consumption + data aggregation energy consumption + data amplification and transmission energy consumption

The cluster whose size is $n_{C H}\left(n_{C H}>2\right)$ has $n_{C H}-1$ normal member. Its cluster head $\mathrm{CH}$ receives $\left(n_{C H}-1\right) * l$ bit and sends $l$ bit at the same time. After data aggregation, data is sent to another cluster head $\mathrm{CH}_{0}\left(\mathrm{CH}_{0}\right.$ may be BS). The energy consumption $E_{\mathrm{CH}-\mathrm{CH}_{0}}$ is calculated as follows.

$$
\begin{aligned}
E_{C H-C H_{0}} & =\operatorname{Re}(C H)+n_{C H} * l * E_{D A}+\operatorname{Tr}(C H) \\
& =\left(n_{C H}-1\right) * l * E_{\text {elec }}+n_{C H} * l * E_{D A}+\alpha * n_{C H} * l *\left[E_{\text {elec }}+\lambda * d_{C H t o C H_{0}}{ }^{\gamma}\right] \\
= & \begin{cases}n_{C H} * l *\left[\alpha * e_{f s} * d_{C H t o C H_{0}}{ }^{2}+E_{D A}\right]+\left[n_{C H}(1+\alpha)-1\right] * l * E_{\text {elec }}, & \text { if } d_{C H t o C H_{0}} \leq d_{0} \\
n_{C H} * l *\left[\alpha * e_{\text {amp }} * d_{C H t o C H_{0}}{ }^{4}+E_{D A}\right]+\left[n_{C H}(1+\alpha)-1\right] * l * E_{\text {elec }}, & \text { if } d_{C H t o C H_{0}}>d_{0}\end{cases}
\end{aligned}
$$

where $\alpha(1>\alpha \geq 0)$ is the compression ratio; $\operatorname{Re}(u)$ represents the energy consumption of node $u$ receiving data, $\operatorname{Tr}(u)$ represents the energy consumption of node $u$ sending data, and $E_{D A}$ represents the energy required for aggregation 1bit data. $d_{v t o u}$ represents the distance from node to node; $d_{u t o C H}$ represents the distance from node $U$ to cluster head $\mathrm{CH} ; d_{\mathrm{CHtoCH}_{0}}$ represents the distance between cluster head $\mathrm{CH}$ and cluster head $\mathrm{CH}_{0}$.

\section{Simulation Experiment}

\subsection{Simulation Environment}

TABLE 2 Simulation Parameters

\begin{tabular}{l|l|l|l|l|l}
\hline Type & Parameter & Value & Type & Parameter & Value \\
\hline Network & Network uniform & From $(0,0)$ to $(100,100)$ & Application & Data packet size & 1000 bytes \\
& Sink & At $(50,140)$ & Broadcast packet size & 250 byptes \\
& Initial energy & $4 \mathrm{~J} /$ battery & & Packet header size & 25 bytes \\
\cline { 1 - 3 } Application & Cluster initial radius & $\boldsymbol{R}_{\mathrm{o}}$ & & Gathering coefficient & 0.8 \\
\hline
\end{tabular}


Matlab is used for simulation experiments. The simulation network model and related parameters are shown in table $1 \sim$ table 2.

\subsection{Results of Simulation}

We use a randomly uniform network with 100 nodes. The related parameters are shown in Table 2. Both the proposed algorithm Alg and HEED are implemented by simulation. In the following figure, the red hollow point ' 0 ' represents living node, solid green dot ' $\bullet$ represents dead node, blue' $*$ 'is an cluster head, ' $\square$ ' representing sink node (or base station). Every star is a cluster (including cluster head, normal member and blue edges representing communication relationship.).

We have made an analysis and comparison from four aspects: 1) living node/round, failed node/round (Fig 3); 2) radius/round figure (Fig 4); 3) equilibrium of cluster distribution (only the case of Algorithm Alg is described; Fig 5); 4) distribution uniformity of living nodes (Fig 6-7). We also compare the rounds of algorithm Alg with HEED in five cases where the first time occurs: first_dead, 10\%_dead, Half_dead, 90\%_dead and All_dead (99\%_dead) (table 3).

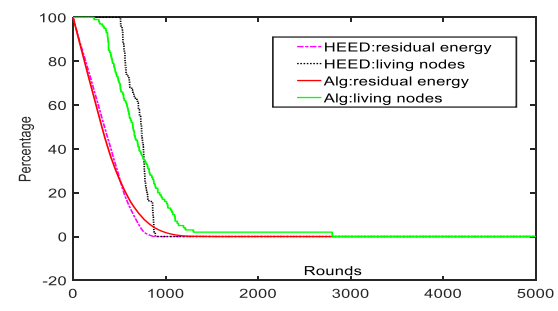

Fig3 Heed and Alg: Percentage of failure nodes, Living nodes, Rounds

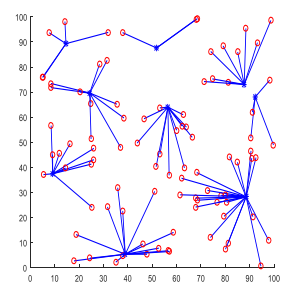

a) Initial network

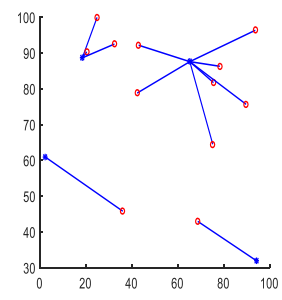

b) most nodes failure

Fig5 Algorithm Alg: Clustering

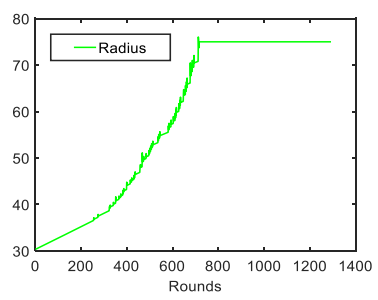

Fig4 Algorithm Alg: Radius/Rounds

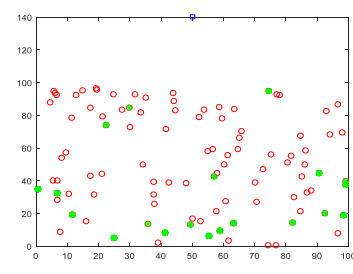

a) 20-node failure

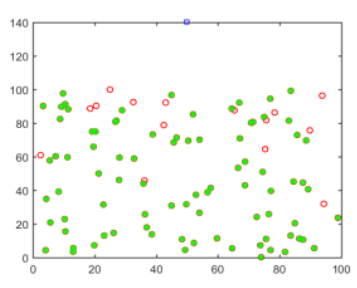

b) most nodes failure

Fig 6 Algorithm Alg: distribution about failure nodes and living nodes

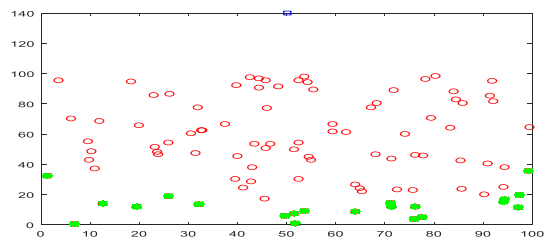

a) 19-node failure

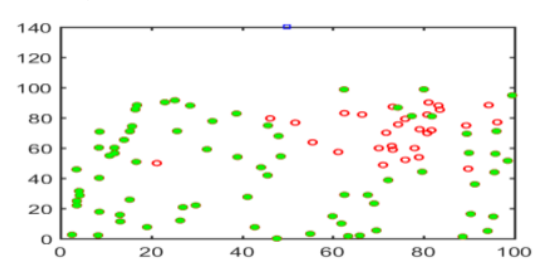

b) most nodes failure

Fig 7 Heed: distribution about failure nodes and living nodes 
TABLE 3 Death Cases vs Rounds

\begin{tabular}{cccccc}
\hline Alg\Death & first_dead & $10 \%$ dead & Half_dead & $90 \%$ dead & All_dead \\
\hline Heed(rounds) & 513 & 539 & 740 & 864 & 889 \\
Alg(rounds) & 255 & 373 & 656 & 1099 & 1293 \\
\hline
\end{tabular}

As shown in Fig 5, the cluster distribution is balanced. Fig 6 and Fig 7 show that algorithm Alg can distribute failure nodes more evenly. Based on Table3 and Fig 3, we can see that the rounds are later when first_dead, 10\%_dead and Half_dead appear in Heed for the first time, but the node failure rate is relatively high in the operation process with this algorithm. The first rounds of first_dead, 10\%_dead and Half_dead appeared in Alg algorithm earlier, but the gap between the corresponding rounds and Heed is gradually narrowed, and the node failure rate of Alg become small during operation. When $90 \%$ of the failed nodes appears, the first round of Alg is later 235 rounds than Heed. Algorithm Alg prolongs the survival time of the network and enables the data to be transferred well in the network as a whole.

\section{Conclusions}

We design and implement routing algorithms Alg in wireless sensor networks with uniform distribution. The rationality of our algorithms is shown. The simulation results show that our algorithms have better performance in energy consumption uniformity.

\section{References}

[1] Piyush Gupta and P. R. Kumar. Critical Power for Asymptotic Connectivity[C]. Proceedings of the 37th IEEE Conference on Decision \& Control.Tampa, Florida USA Dec. 1998, 1106-1110.

[2] Sanjay Shakkottai, R.Srikant, NessShroff. Unreliable Sensor Grids: Coverage, Connectivity and Diameter[C]. Proc of the 22nd Annual Joint Conference of the IEEE Computer and Communications Societies. San Francisco, IEEE, 2003, 1073-1083.

[3] Heinzelma, A. Chandrakasan and H. Balakrishnan. Energy-Efficient Communication Protocol for Wireless Microsensor Networks. Proceedings of the 33rd Hawaii International Conference on System Sciences, Jan. 2000. (Proc. 33rd Hawaii Int'l. Conf. Sys. Sci., Jan. 2000)

[4] W.B. Heinzelman, A.P. Chandrakasan, H. Balakrishnan. An application specific protocol architecture for wireless microsensor networks, IEEE Trans. Wirel. Commun. 1, Oct. 2002, 4, 660-670.

[5] G. Smaragdakis, I. Matta, A. Bestavros. SEP: A Stable Election Protocol for clustered heterogeneous wireless sensor networks. Second International Workshop on Sensor and Actor Network Protocols and Applications (SANPA), 2004, 1-11.

[6] Dongmei Xing. Energy Consumption Balancing Algorithms on Optimizing Dynamic Topology in Wsns. Frontiers in Artificial Intelligence and Applications, Volume 320: Fuzzy and Data Mining VProceedings of FDSM 2019, 975-983. DOI:10.3233/FAIA 190273.

[7] Vishal Kumar Arora (Research Scholar), Vishal Sharma, Monika Sachdeva. A survey on LEACH and other's routing protocols in wireless sensor network, Optik 127 (2016), 6590-6600.

[8] O. Younis, S. Fahmy. HEED: A Hybrid, Energy-Efficient, Distributed Clustering Approach for Adhoc Sensor Networks, IEEE Transactions on Mobile Computing, 2004 , 3 (4), 366-379.

[9] Kuang ZheJun. Research on energy saving strategies for wireless sensor networks (Thesis, in Chinese). Doctoral thesis of Jilin university, China. May 2014.

[10] PrasenjitChanak, IndrajitBanerjee, HafizurRahaman. Load management scheme for energy holes reduction in wireless sensor networks[J]. Computers and Electrical Engineering, 48 (2015), 343-357.

[11] Tarunpreet Bhatia,Simmi Kansal, Whivani Geol, A.K. verma . A genetic algorithm based distanceaware routing protocol for wireless senor networks. Computers and Electrical Engineering, 56 (2016), 441-455. http://dx.doi.org/10.1016/ j.compeleceng.2016.09.016.

[12] Tianshu Wang, Gongxuan Zhang, Xichen Yang, Ahmadreza Vajdi. Genetic algorithm for energyefficient clustering and routing in wireless sensor networks. The Journal of Systems and Software, 146 (2018), 196-214. https: //doi.org /10.1016/j.jss.2018.09.067. 
[13] K. Thangaramya, K. Kulothungan, R. Logambigai, M. Selvi, Sannasi Ganapathy, A. Kannan. Energy aware cluster and neuro-fuzzy based routing algorithm for wireless sensor networks in IoT. Computer Networks, 151 (2019), 211-223. https://doi.org/10.1016/j.comnet.2019.01.024.

[14] Ngoc-Tu Nguyen, Bing-Hong Liu, Van-Trung Pham, Yi-Sheng Luo. On maximizing the lifetime for data aggregation in wireless sensor networks using virtual data aggregation trees. Computer Networks, 105 (2016), 99-110. http://dx.doi.org/10.1016/j.comnet.2016.05.022.

[15] Anuradha Pughat, Vidushi Sharma. Performance analysis of an improved dynamic power management model in wireless sensor node. Digital Communications and Networks, 3 (2017), 19-29.

[16] Mohit Sajwan, Devashish Gosain, Ajay K. Sharma. Hybrid energy-efficient multi-path routing for wireless sensor networks. Computers and Electrical Engineering, 67 (2018), 96-113. https: //doi.org/10.1016/ j.compeleceng. 2018.03.018.

[17] Ansam Ennaciri, Mohammed Erritali, Jamaa Bengourram. Load Balancing Protocol (EESAA) to improve Quality of Service in Wireless sensor network. Procedia Computer Science, 151 (2019), $1140-1145$.

[18] ISurjit Singh, Rajeev Mohan Sharma. Optimization Techniques in Wireless Sensor Networks. CTCS '16, March 04-05, 2016, Udaipur, India. DOI: http://dx.doi.org /10.1145/2905055.2905200.

[19] Maryam Bandari, Robert Simon, and Hakan Aydin. 2017. DMS based energy optimizations for clustered WSNs. ACM Trans. Embed. Comput. Syst. 3(16), Article 86 (April 2017), 28 pages. DOI: http://dx.doi.org/ 10.1145/2998179

\section{Appendix}

\section{Algorithm Alg}

\section{Initialize}

1. Compute $f_{R}, f_{P}$ and $R$; Determine the neighboring set $S_{n b r}$ of each node $S_{n b r} \leftarrow\{v: v$ lies within my cluster range $\}$ 。

2. Compute and broadcast cost to $v \in S_{n b r}$

3.

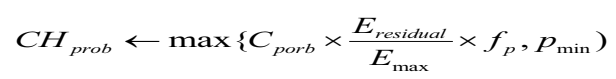

4. is_final_ $C H \leftarrow F A L S E$

II. Repeat

1. If $\left(\left(S_{C H} \leftarrow\{v: v\right.\right.$ is a cluster hand $\left.\left.\}\right) \neq \varnothing\right)$

2. $\quad$ my_cluster_head $\leftarrow$ least_ $\cos t\left(S_{C H}\right)$

3. If $\left(\mathrm{CH}_{\text {prob }}=1\right)$

4. Cluster_head_msg(NodeID, final_CH, $\cos t)$

5. $\quad$ is $_{-}$final_ $C H \leftarrow T R U E$, compute $R$ and $d$

6. else

7. If $m y \_$cluster_head $=$NodeID

8. Cluster_head_msg(NodeID,tentive_CH, $\cos t)$

9. else If $\operatorname{Random}(0,1) \leq \mathrm{CH}_{\text {prob }}$

10. Cluster_head_msg(NodeID,tentive_CH, $\cos t)$

11. $\mathrm{CH}_{\text {previous }} \leftarrow \mathrm{CH}_{\text {prob }}$

12. $C H_{\text {prob }} \leftarrow \min \left\{C_{\text {porb }} \times 2,1\right)$

13. Until $C H_{\text {previous }}=1$

III. Confirm

1. If (is_final_CH=FALSE)

2. If $\left(S_{C H} \leftarrow\{v: v\right.$ is a cluster head $\left.\}\right) \neq \varnothing$ 
4.

$$
\text { join_cluster(cluster_head_ID,NodeID) }
$$

5. else Cluster_head_msg(NodeID, final_CH, $\cos t)$,

6. else Cluster_head_msg(NodeID, final_CH, cos $t)$

IV. Adjust

1. If $\left(\left|S_{n b r}\right|=1\right)$ and (is_final_ $C H=T R U E$ )

2. $S_{C H} \leftarrow S_{C H}-\{N o d e I D\}$, is _final_CH=FALSE

3. my_cluster_head $\leftarrow$ least_cost $\left(\mathrm{S}_{\mathrm{CH}}\right)$

4. join_cluster(cluster_head_ID, NodeID)

5. If ( $\left|S_{n b r}\right|=2$ ) and (is_final_ $C H=T R U E$ )

6. $S_{C H} \leftarrow S_{C H}-\{N o d e I D\}$, is final_ $\mathrm{CH}=\mathrm{NNON}{ }_{-} \mathrm{CH}$

7. my_cluster_head $\leftarrow$ least_cost $\left(\mathrm{S}_{\mathrm{CH}}\right)$

8. join_cluster(cluster_head_ID, NodeID) 